\title{
On infinite-horizon probabilistic properties and stochastic bisimulation functions
}

\author{
Ilya Tkachev and Alessandro Abate
}

\begin{abstract}
This work investigates infinite-horizon properties over discrete-time stochastic models with continuous state spaces. The focus is on understanding how the structural features of a model (e.g., the presence of absorbing sets) affect the values of these properties and relate to their uniqueness. Furthermore, we argue that the investigation of these features can lead to approximation bounds for the value of such properties, as well as to improvements on their computation. The article employs the presented results to find a stochastic bisimulation function of two processes.
\end{abstract}

\section{INTRODUCTION}

The analysis of general state-space stochastic models has recently witnessed substantial efforts towards the use of formal verification techniques [2], [7]. Recent work has drawn formal connections between the solution of dynamic programming problems, which constitutes the underlying computation engine for a number of studies (such as (probabilistic) reachability, invariance, and reach-avoid), and the verification of related specifications in PCTL logic [3], [13] (until properties). Along similar lines, control synthesis problems have been tackled with approaches that can be related to probabilistic model checking [4]. These research efforts have focused on models as general as stochastic hybrid systems, namely hybrid models with stochastic continuous dynamics as well as probabilistic jumps and resets.

There are two main approaches to study classes of infinitetime properties for general state-space processes: 1) as the limit of the iteration of certain operators, or 2) as the solution of integral (Bellman-like) equations. Understanding the properties and the computations related to the solution of infinite-horizon properties [1] has lead to a few early results [13], such as lack of uniqueness or triviality of solutions.

This work looks at discrete-time, general state-space models (its results can be for instance applied to stochastic hybrid systems - dtSHS - as in [2], [4]) and has three main objectives. First, it seeks to shed new light over the existence of unique or trivial solutions of infinite-time properties such as probabilistic invariance and reachability. Second, it puts forward a technique to ease the computation of infinite-time invariance, by hierarchically breaking down this property into two subproblems and by approximating their solution. Third, it exhibits the application of the above results to the problem

This work is supported by the European Commission under the MoVeS project FP7-ICT-2009-257005, by the European Commission under Marie Curie grant MANTRAS 249295, and by NWO under VENI grant 016.103.020.

The authors are with the Delft Center for Systems \& Control, Technische Universiteit Delft - Delft University of Technology, The Netherlands. Emails: $\{$ i.tkachev, a. abate\}@tudelft.nl of finding a stochastic bisimulation function (SBF) of two processes [9].

The first problem (Section III) is elaborated by looking at the presence of absorbing sets [11] over regions of the continuous state space. These sets turn out to play a critical role, both formally and computationally. As for the second problem, in Section IV we propose a lower bound for infinite-time probabilistic invariance by expressing this property via a finite-time reach-avoid plus an infinitetime invariance around absorbing sets on the state space. An example is proposed in Section IV-D to elucidate the results. With regards to the third problem (seeking a SBF of two given processes), we put forward in Section $\mathrm{V}$ an approach that is distinguished from [9] in that we do not raise assumptions (e.g. linearity, stability) on the dynamics of the two processes under study - a computational study is developed in Section V-A to help explain the results. Proofs are omitted from this manuscript due to space constraints.

\section{PReliminaries}

Consider a discrete-time homogeneous Markov process $X$ on the Borel space $\mathscr{X}$ together with a Borel $\sigma$ algebra $\mathscr{B}(\mathscr{X})$ (see e.g. [8]). We define the stochastic basis $(\Omega, \mathscr{F}, \mathbb{F}, \mathrm{P})$, where $\mathbb{F}=\left\{\mathscr{F}_{n}\right\}_{n \geq 0}$ and $\mathscr{F}_{n}=\left\{X_{k}, 0 \leq k \leq n\right\}$ [6]. For each distribution of the initial state denoted as $\pi$, we define the probability measure $\mathrm{P}_{\pi}$ such that

$$
\mathrm{P}_{\pi}\left\{X_{0} \in A\right\}=\pi(A)
$$

for all $A \in \mathscr{B}(\mathscr{X})$. An expectation with respect to this measure is denoted as $\mathrm{E}_{\pi}$. Similarly, we denote by $\mathrm{P}_{x}(\cdot)=$ $\mathrm{P}\left(\cdot \mid X_{0}=x\right)$ and the corresponding expectation by $\mathrm{E}_{x}$. The transition kernel associated to the process $X$ is a measure on $(\mathscr{X}, B(\mathscr{X}))$ for each $x \in \mathscr{X}$ given by $T(A \mid x)=\mathrm{P}_{x}\left\{X_{1} \in A\right\}$.

For a measurable function $f: \mathscr{X} \rightarrow \mathbb{R}$ let us put $f^{-}(x)=$ $\max \{0,-f(x)\}$. We say that $f \in \mathscr{L}$ if $\mathrm{E}_{x} f^{-}\left(X_{1}\right)<\infty$ for any $x \in \mathscr{X}$. For $f \in \mathscr{L}$ a transition operator $\mathscr{P}$ and an excessive operator $\mathscr{Q}$ are defined as follows:

$$
\begin{aligned}
& \mathscr{P} f(x)=\mathrm{E}_{x}\left[f\left(X_{1}\right)\right], \\
& \mathscr{Q} f(x)=\max \{f(x), \mathscr{P} f(x)\} .
\end{aligned}
$$

With $\mathscr{C}(B)$ we define the class of real-valued functions that are continuous and bounded on $B \in \mathscr{B}(\mathscr{X})$ and for a measurable function $g: \mathscr{X} \rightarrow \mathbb{R}$ and $\delta \in \mathbb{R}$ we define the superlevel set $\mathscr{X}_{g}(\delta)=\{x \in \mathscr{X}: g(x)>\delta\}$. 


\section{PROBABILISTIC REACHABILITY AND INVARIANCE}

\section{A. Reachability as an optimal stopping problem}

On the set $\Omega$ we define the following events for any $N \geq 0$ and $A \in \mathscr{B}(\mathscr{X})$ :

$$
\begin{aligned}
r_{N}(A) & =\left\{\exists n \in[0, N]: X_{n}(\omega) \in A\right\}, \\
r_{A} & =\left\{\exists n \geq 0: X_{n}(\omega) \in A\right\} .
\end{aligned}
$$

We formally show the connection between calculating the probability of these events and solving an optimal stopping problem. Let us first define a stopping time [6].

Definition 1. A random variable $\tau$ is called a stopping time for the process $X$ if for all $n \geq 0$ the event $\{\tau \leq n\} \in \mathscr{F}_{n}$ and $\mathrm{P}\{\tau<\infty\}=1$. For any subset $A \in \mathscr{B}(\mathscr{X})$ we also denote $\tau_{A}=\inf \left\{n \geq 0: X_{n} \in A\right\}$ as a first entry time for the set $A$.

We introduce the following proposition, also discussed in [5, Prop.6, p. 6176].

Proposition 1. For any $N \geq 0$ and $A \in \mathscr{B}(\mathscr{X})$ we have

$$
\mathrm{P}\left[r_{N}(A)\right]=\sup _{\tau \leq N} \mathrm{E}\left[\mathbb{I}_{A}\left(X_{\tau}\right)\right]
$$

where $\mathbb{I}_{A}(\cdot): \mathscr{X} \rightarrow\{0,1\}$ is an indicator function of the set $A$ and $\tau$ is a stopping time. Moreover, the stopping time $\tau^{*}=\tau_{A} \wedge N=\min \left\{\tau_{A}, N\right\}$ is an optimal stopping time in the optimal stopping problem (2).

Corollary 1. For any $A \in \mathscr{B}(\mathscr{X})$,

$$
\mathrm{P}\left[r_{A}\right]=\lim _{N \rightarrow \infty} \mathrm{P}\left[r_{N}(A)\right] .
$$

Note that these facts hold over arbitrary probability measures, in particular over $\mathrm{P}_{x}$, for any $x \in \mathscr{X}$.

The characterization of probabilistic reachability as an optimal stopping problem leads to the following result, which indicates a method to compute this quantity and suggests a different perspective on [4].

Theorem 1. Let $A \in \mathscr{B}(\mathscr{X})$ and $n \in \mathbb{N}$. Then

$$
\begin{aligned}
\mathrm{P}_{x}\left[r_{n}(A)\right] & =\mathscr{Q}^{n} \mathbb{I}_{A}(x), \\
\mathrm{P}_{x}[r(A)] & =\lim _{n \rightarrow \infty} \mathscr{Q}^{n} \mathbb{I}_{A}(x),
\end{aligned}
$$

for all $x \in \mathscr{X}$, where as in (1), $\mathscr{Q} f(x)=\max \{f(x), \mathscr{P} f(x)\}$.

\section{B. Probabilistic invariance - definition}

Probabilistic invariance is dual to the problem of stochastic reachability. It was studied in [2], assuming the process $X$ is a realization of a dtSHS. For a given set $A \in \mathscr{B}(\mathscr{X})$ and a time horizon $n \in \mathbb{N}$ we define corresponding value functions

$$
u_{n}(x ; A)=\mathrm{P}_{x}\left\{X_{i} \in A \text { for all } 0 \leq i \leq n\right\}
$$

and

$$
v_{n}(x ; A)=\mathrm{P}_{x}\left[r_{n}(A)\right] .
$$

Clearly we have the following identity

$$
u_{n}(x ; A)=1-v_{n}\left(x ; A^{c}\right),
$$

where $A^{c}:=\mathscr{X} \backslash A$.
On the other hand we know that (see e.g. [14, Th.1, p.28])

$$
v_{n}\left(x ; A^{c}\right)=\max \left\{\mathbb{I}_{A^{c}}(x), \mathscr{P} v_{n-1}\left(x ; A^{c}\right)\right\},
$$

where $v_{0}\left(x ; A^{c}\right)=\mathbb{I}_{A^{c}}(x)$ and hence

$$
u_{n}(x ; A)=\min \left\{\mathbb{I}_{A}(x), \mathscr{P} u_{n-1}(x ; A)\right\}
$$

(compare e.g. [2, Prop.1, p.4]). We can also provide another way to calculate the functions $u_{n}$ : note that, from (4),

$$
u_{n}(x ; A)=\left\{\begin{aligned}
\mathbb{I}_{A}(x), & \text { if } x \in A^{c} \\
\mathscr{P} u_{n-1}(x ; A), & \text { if } x \in A
\end{aligned}\right.
$$

hence for all $n \geq 1$ we have

$$
u_{n}(x ; A)=\mathbb{I}_{A}(x) \mathscr{P} u_{n-1}(x ; A)=\mathbb{I}_{A}(x) \int_{\mathscr{X}} u_{n-1}(y ; A) T(d y \mid x),
$$

where $T$ is the transition kernel associated to the discrete time homogenous Markov process $X$ and $u_{0}(x ; A)=\mathbb{I}_{A}(x)$. Let us introduce the operator $\mathscr{I}_{A}$ which is defined for functions $f \in \mathscr{L}$ :

$$
\mathscr{I}_{A} f(x)=\mathbb{I}_{A}(x) \int_{\mathscr{X}} f(y) T(d y \mid x) .
$$

With regards to the infinite horizon invariance, let us introduce the event $\left\{X_{n} \in A\right.$ for all $\left.n \geq 0\right\}$. It can be easily seen that

$$
\begin{aligned}
& \mathrm{P}_{x}\left\{X_{n} \in A \text { for all } n \geq 0\right\}=1-\mathrm{P}_{x}\left[r\left(A^{c}\right)\right], \text { thus } \\
& \mathrm{P}_{x}\left\{X_{n} \in A \text { for all } n \geq 0\right\}=u(x ; A):=\lim _{n \rightarrow \infty} u_{n}(x ; A),
\end{aligned}
$$

where the limit exists pointwise for all $x \in \mathscr{X}$ (cf. [1, Th.3, p.263]). We denote $v\left(x ; A^{c}\right)=\mathrm{P}_{x}\left[r\left(A^{c}\right)\right]=\lim _{n \rightarrow \infty} v_{n}\left(x ; A^{c}\right)$.

\section{Trivial solutions of probabilistic invariance}

Theorem 2. Suppose that for some $N$ we have

$$
u_{N}(x ; A) \leq \alpha<1
$$

for all $x \in A$, then $u(x ; A) \equiv 0$.

This fact can be exploited in the computation of $u(x ; A)$, and leads us to the question of whether there will be an $N$ such that $\sup _{x \in A} u_{N}(x ; A)<1$. To answer this question we introduce the notion of an absorbing set [11, Def.IV, p.91].

Definition 2. A non-empty set $B \in \mathscr{B}(\mathscr{X})$ is called absorbing if for all $x \in B$ the following identity holds

$$
T(B \mid x)=1 .
$$

The absorbing set $B \subseteq A$ is called an absorbing subset of $A$. For the set $A \in \mathscr{B}(\mathscr{X})$ the set $B$ is the largest absorbing subset of $A$ if for any absorbing set $B^{\prime} \subseteq A$ holds $B^{\prime} \subseteq B$.

Definition 3. The transition kernel $T$ is called weakly continuous [8] if $\mathscr{P} f \in \mathscr{C}(\mathscr{X})$ whenever $f \in \mathscr{C}(\mathscr{X})$. We write then $T \in \mathscr{C}_{w}(\mathscr{X})$. 


\section{Invariance and absorbing sets}

Consider the following family of sets:

$$
A_{n}=\left\{x \in A: u_{n}(x ; A)=1\right\} .
$$

Here we note that $A=A_{0}$ and $A_{1} \subseteq A_{0}$. Define the following limit set

$$
A_{\infty}=\bigcap_{n=0}^{\infty} A_{n} .
$$

Lemma 1. For all $n \geq 0$ we have

$$
A_{n+1}(x)=\left\{x: T\left(A_{n} \mid x\right)=1\right\} .
$$

and $A_{n+1} \subseteq A_{n}$.

Theorem 3. Let $A \in \mathscr{B}(\mathscr{X})$ be a compact set and $T \in$ $\mathscr{C}_{w}(\mathscr{X})$. Then

$$
u(x ; A)=0
$$

for all $x \in \mathscr{X}$ if and only if there are no absorbing subsets of $A$. Moreover, if there is an absorbing subset of $A$ then $A_{\infty}$, as in Lemma 1, is the largest absorbing subset of $A$.

Remark 1 (On the computation of (6)). In some verification problems it is relevant to calculate an absorbing subset of a given set A, or to overapproximate it [3]. Using the monotonicity of the procedure in (6) we conclude that if $A_{n}^{\prime} \supseteq A_{n}$ is an overapproximation of the set $A_{n}$ and

$$
A_{n+1}^{\prime} \supseteq\left\{x: T\left(A_{n}^{\prime} \mid x\right)=1\right\}
$$

then $A_{n+1}^{\prime} \supseteq A_{n+1}$. The right-hand side in (7) can be precisely overapproximated by means of a state space discretization algorithm [2] (the requirement on Lipshitz continuity in [2] also verifies the continuity assumption in Definition 3).

\section{REACH-AVOID PROBLEM}

\section{A. Bounds for infinite horizon reach-avoid problem}

For the sets $A, B \in \mathscr{B}(\mathscr{X})$ such that $A \cap B=\emptyset$ we define the reach-avoid probability as follows

$$
w(x ; A, B)=\mathrm{P}_{x}\left\{\exists n>0: X_{k} \in A \forall k<n, X_{n} \in B \text { or } X_{0} \in B\right\} .
$$

The set $B$ is a target (reach) set, the set $A$ is a set of legal states and the set $\mathscr{X} \backslash(A \cup B)$ is an illegal (avoid) set.

To calculate the reach-avoid probability value in $w(x ; A, B)$ we set up a procedure, as described in [13] for the unbounded-until specification, which is a specification in modal logic that corresponds to the reach-avoid problem.

Let us define the operator

$$
\mathscr{R}_{A, B} f(x)=\mathbb{I}_{B}(x)+\mathbb{I}_{A}(x) \int_{\mathscr{X}} f(y) T(d y \mid x) .
$$

If we denote $w_{0}(x ; A, B)=\mathbb{I}_{B}(x)$, then for $n \geq 0$ define

$$
w_{n+1}(x ; A, B)=\mathscr{R}_{A, B} w_{n}(x ; A, B) .
$$

This sequence in non-decreasing and bounded from above by 1 . Moreover,

$$
w(x ; A, B)=\lim _{n \rightarrow \infty} w_{n}(x ; A, B),
$$

where the limit is taken pointwise (see [13, (3.7)]).
The following theorem provides strict bounds for the convergence in (8).

Theorem 4. Suppose that for some $N$ we have

$$
u_{N}(x ; A) \leq \alpha<1
$$

for all $x \in A$. Then, for any $n$,

$$
w(x ; A, B)-w_{n}(x ; A, B) \leq \frac{N \alpha\left\lfloor\frac{n}{N}\right\rfloor}{1-\alpha} .
$$

Remark 2. To calculate the finite-horizon quantity $w_{n}(x ; A, B)$ one can use the discretization technique and formal bounds provided in [2].

B. Decomposition of the infinite-horizon invariance probability

For the reach-avoid problem we derived computational bounds for the case when the solution in (8) may be nontrivial. The following theorem shows how to bound invariance on the infinite horizon by two different problems: using a reach-avoid value function, and an invariance problem.

Theorem 5. Let $T \in \mathscr{C}_{w}(\mathscr{X})$ and sets $A, B \in \mathscr{B}(\mathscr{X})$ be such that $B \subseteq A$ and $A^{\prime} \backslash B$ is a compact set without absorbing subsets. Then for all $x \in A^{\prime}$ we have

$$
u(x ; A) \geq w(x ; A, B) \psi(A, B),
$$

where

$$
\psi(A, B)=\inf _{x \in B} u(x ; A) .
$$

Note that due to the monotonicity of the sequence $w_{n}\left(x ; A^{\prime}, B\right)$ we have

$$
u(x ; A) \geq w_{n}(x ; A, B) \psi(A, B),
$$

for all $n \geq 0$. Thus, the main question is how to find $\psi(A, B)$ or how to find bounds for this quantity, which we answer in the next section. Notice that the definition of $\psi(A, B)$ focuses on points in set $B \subseteq A$ : this directly leads to computational advantages when calculating this quantity over $u(x ; A)$.

\section{Bounds via local Doob's inequality}

We leverage the following Doob's inequality [6], [12]: if for some fixed $x \in \mathscr{X}$ the process $g\left(X_{n}\right)$ is a non-negative $\mathrm{P}_{x}$-supermartingale for all $n \leq N$, then for all $\delta>0$ it holds that

$$
\mathrm{P}_{x}\left\{\sup _{0 \leq n \leq N} g\left(X_{n}\right) \geq \delta\right\} \leq \frac{1}{\delta} \sup _{0 \leq n \leq N} \mathrm{E}_{x}\left[g\left(X_{n}\right)\right]=\frac{g(x)}{\delta} .
$$

Definition 4. A measurable function $g \in \mathscr{L}$ is said to be an excessive function (for a process $X$ or with respect to an operator $\mathscr{P})$, if

$$
\mathscr{P} g(x) \leq g(x) .
$$

for all $x \in \mathscr{X}$. Let us call the function $g$ excessive in $B \in \mathscr{B}(\mathscr{X})$ if for all $x \in B$ it holds that $\mathscr{P} g(x) \leq g(x)$. The maximal excessive set for the function $g$ is the set

$$
\mathscr{E}_{g}=\{x \in \mathscr{X}: \mathscr{P} g(x) \leq g(x)\} .
$$


Lemma 2. [12, (1.2.40), p. 18] The function $g$ is excessive if and only if $\left(g\left(X_{n}\right)\right)_{n \geq 0}$ is a $\mathrm{P}_{x}$-supermartingale for all $x \in \mathscr{X}$.

Often inequality (12) requires conservative assumptions on function $g$ : indeed, the previous lemma shows that inequality (12) is only satisfied for excessive functions $g$. [10] presents a relaxation of Doob's inequality for functions that are excessive only locally (see bottom of Def. 4).

Theorem 6. [10, Theorem 12, p. 71$]$ If $g$ is a measurable and non-negative function such that $\mathscr{X}_{g}^{c}(\delta) \subseteq \mathscr{E}_{g}$ for some $\delta>0$ then

$$
\mathrm{P}_{x}\left\{\sup _{n \geq 0} g\left(X_{n}\right) \geq \delta\right\} \leq \frac{g(x)}{\delta} .
$$

for all $x \in \mathscr{X}_{g}^{c}(\delta)$.

\section{Decomposition of probabilistic invariance: an example}

This example displays the use of the bounds in Sections IV-B and IV-C over a linear Gaussian discrete-time system. Let $\mathscr{X}=\mathbb{R}^{m}$ and $M$ is a symmetric positive semidefinite matrix. Let us consider the following Markov process $X$ :

$$
X_{n+1}-X_{n}=\mu\left(X_{n}\right)+\sigma\left(X_{n}\right) \xi_{n},
$$

where $\mu: \mathbb{R}^{m} \rightarrow \mathbb{R}^{m}, \sigma: \mathbb{R}^{m} \rightarrow \mathbb{R}^{m \times k}$ and $\xi_{n}$ is a standard $k$ dimensional Gaussian random vector, $\xi_{n^{\prime}} \perp \xi_{n^{\prime \prime}}$ for all $n^{\prime} \neq$ $n^{\prime \prime}$. We select a function $g$ with a quadratic form $g(x)=$ ${ }^{T} M x$. Suppose that for some $\delta^{\prime}>0$ the following inequality holds, for all $x \in \mathscr{X}_{g}^{c}\left(\delta^{\prime}\right)$ :

$$
\mu^{T}(x) M \mu(x)+2 \mu^{T}(x) M x+\operatorname{Tr}\left(\sigma^{T}(x) M \sigma(x)\right) \leq 0 .
$$

Then, by application of the inequality in (12), for all $\delta \leq \delta^{\prime}$ we have

$$
v\left(x ; \mathscr{X}_{g}(\delta)\right) \leq \frac{x^{T} M x}{\delta} \text { i.e. } u\left(x ; \mathscr{X}_{g}^{c}(\delta)\right) \geq 1-\frac{x^{T} M x}{\delta} .
$$

We show how this bounds can be improved in the case when $M$ is positive definite, by leveraging the decomposition technique in Section IV-B. We select sets $A=\mathscr{X}_{g}^{c}(\delta)$ and $B=\{x \in \mathscr{X} \mid g(x)<\varepsilon\}$ for $\varepsilon<\delta$. By Theorem 6, we obtain

$$
\psi(A, B) \geq 1-\frac{\varepsilon}{\delta} \text {. }
$$

Furthermore, we can use the inequality in (10), obtaining

$$
u\left(x ; A^{\prime}\right) \geq w(x ; A, B)\left(1-\frac{\varepsilon}{\delta}\right) .
$$

Finally, the term $w(x ; A, B)$ can be calculated with any accuracy since $A=A^{\prime} \backslash B$ does not contain the origin, which is the only absorbing subset of $\mathscr{X}$, thus the conditions of Theorem 4 are satisfied.

\section{E. Probabilistic invariance over finite state spaces}

In the previous section we have described how to find bounds for $\psi(A, B)$ in the case when $A$ and $B$ are sublevel sets of some locally excessive function $g$. In general one can apply discretization techniques [2] to estimate $\psi(A, B)$ by working with a finite space Markov chain approximation. With focus on this latter class of probabilistic models, we present next an explicit solution for the infinite horizon invariance problem for a finite space Markov chain.

Consider a Markov chain $Y$ with a state space $\mathscr{Y}=$ $\left\{y_{i}\right\}_{i=1}^{N}$. Without loss of generality we put $y_{i}=i$ for $1 \leq i \leq N$. Now, $p_{i j}=\mathrm{P}\left(Y_{1}=j \mid Y_{0}=i\right)$ form a stochastic matrix $\mathrm{P}$. For any given set $A \subseteq \mathscr{Y}$ we consider a vector u, which components

$$
\mathrm{u}_{i}=\mathrm{P}\left\{Y_{n} \in A \text { for all } n \geq 0 \mid Y_{0}=i\right\}
$$

denote infinite-horizon invariance probabilities. Vector $u$ has to be a solution of the following equation

$$
u=\mathrm{D}(A) \cdot \mathrm{Pu},
$$

where $\mathrm{D}(A)$ is the diagonal matrix such that $D_{i i}(A)=1$ if and only if $i \in A$. The following holds:

Proposition 2. Equation (14) has a unique solution $u=0$ if and only if there is no an absorbing subset of $A$.

Let us now look to the instance where the chain admits an absorbing subset $A^{\prime} \subseteq A$, and let us also suppose that $A^{\prime}$ is the largest absorbing subset of $A$, so $A \backslash A^{\prime}$ has no absorbing subsets. Without loss of generality suppose that $A^{\prime}=[1, m]=1,2, \ldots, m$ and $A=[1, n]$ where $m \leq n \leq N$ since it is always possible to sort the elements of set $\mathscr{Y}$ in order to obtain this form. Clearly

$$
\mathrm{u}_{i}=\left\{\begin{array}{l}
1, \text { for } 1 \leq i \leq m, \\
0, \text { for } n+1 \leq i \leq N .
\end{array}\right.
$$

We rewrite the equation (14) in the following form

$$
\left(\mathrm{I}_{N}-\mathrm{D}(A) \cdot \mathrm{P}\right) \mathrm{u}=\mathrm{Qu}=0,
$$

where we denote an identity matrix of dimension $N$ with $I_{N}$. Obviously $\operatorname{det}(\mathrm{Q})=0$, so 1 is an eigenvalue of the matrix $\mathrm{D}(A) \cdot \mathrm{p}$. We put $\left(q_{i j}\right)_{i, j=1}^{N}$ to be elements of the matrix $\mathrm{Q}$. As we discussed, only the elements $\mathrm{u}_{i}$ for $m+1 \leq i \leq n$ are unknown. We can compute them from the equation $\mathrm{Qu}=0$ :

$$
\sum_{j=1}^{m} q_{i j}+\sum_{j=m+1}^{n} q_{i j} u_{j}=0
$$

for $m+1 \leq i \leq n$. This equation has a unique solution if the coefficients $q_{i j}$ for $m+1 \leq i, j \leq n$ form a matrix with nonzero determinant. Indeed, they form the $[m+1, n] \times[m+1, n]$ block of the matrix $\mathrm{Q}$, which we denote as $\mathrm{Q}^{\prime}$. Let us show that the determinant is non-zero by contradiction. Assume the contrary, $\operatorname{det}\left(\mathrm{I}_{n-m}-\mathrm{Q}^{\prime}\right)=0$ which implies that $\operatorname{det}\left(\mathrm{I}_{N}-\right.$ $\mathrm{D}([m+1, n]) \cdot \mathrm{P})=0$.

But this is equivalent to the statement that the equation $\mathrm{u}^{\prime}=\mathrm{D}([m+1, n]) \cdot \mathrm{Pu}^{\prime}$ has non-unique solution, which is not true since $A^{\prime}$ is a largest absorbing subset of $A$ and hence, the interval $[m+1, n]$ of elements of $\mathscr{Y}$ does not contain absorbing subsets.

In conclusion, we can summarize the discussion above with the following proposition.

Proposition 3. In the presence of an absorbing set $A^{\prime}$ the solution the invariance problem (13) over $A, A^{\prime} \subseteq A$, is a nontrivial solution of (14) and is uniquely defined by (15)-(16). 


\section{Stochastic Bisimulation Functions}

Consider a stochastic model $\mathscr{S}^{1}$ with associated discretetime, (time-)homogeneous strong Markov process $X^{1}$, and a second model denoted by $\mathscr{S}^{2}$ and $X^{2}$. ( $\mathscr{S}^{1}$ may be thought of as a concrete model, whereas $\mathscr{S}^{2}$ as its abstraction.) We are interested in quantifying "how close" $\mathscr{S}^{1}$ and $\mathscr{S}^{2}$ are, which can be done by selecting a function $g$ inducing a metric over the distance between their trajectories [9]. The quality of the abstraction is characterized, over a finite and an infinite time horizon, by the following quantities:

$$
V_{\delta}^{N}(x)=\mathrm{P}_{x}\left\{\sup _{0 \leq n \leq N} g\left(X_{n}\right) \geq \delta\right\}
$$

and

$$
V_{\delta}(x)=\mathrm{P}_{x}\left\{\sup _{n \geq 0} g\left(X_{n}\right) \geq \delta\right\},
$$

where $g$ is a measurable non-negative function for $X=$ $\left(X^{1}, X^{2}\right)$, a Markov process for the joint system $\mathscr{S}=$ $\left(\mathscr{S}^{1}, \mathscr{S}^{2}\right)$. For a finite horizon $N$, it can be noticed that

$$
V_{\delta}^{N}(x)=\mathrm{P}_{x}\left[r_{N}\left(\mathscr{X}_{g}(\delta)\right)\right],
$$

thus $V_{\delta}^{N}(x)$ can be calculated using (3).

Next, we focus on the infinite horizon case, where

$$
V_{\delta}(x)=\mathrm{P}_{x}\left[r\left(\mathscr{X}_{g}(\boldsymbol{\delta})\right)\right]=v\left(x ; \mathscr{X}_{g}(\boldsymbol{\delta})\right) .
$$

However, as discussed, this latter quantity is in general difficult to precisely quantify. Alternatively, one can exploit the use of a stochastic bisimulation function (SBF), as developed for continuous-time models in [9].

Definition 5. Let the measurable function $\varphi: \mathscr{X} \rightarrow \mathbb{R}_{+}$ satisfy the following conditions

1) $\left(\varphi\left(X_{n}\right)\right)_{n \geq 0}$ is a $\mathrm{P}_{x}$-supermartingale for any fixed $x \in$ $\mathscr{X}$

2) $\varphi(x) \geq g(x)$ for all $x \in \mathscr{X}$.

Then $\varphi$ is an SBF for the function $g$ (with respect to the process $X$ ).

If $\varphi$ is an SBF of $g$ then

$$
\mathrm{P}_{x}\left\{\sup _{n \geq 0} g\left(X_{n}\right) \geq \delta\right\} \leq \mathrm{P}_{x}\left\{\sup _{n \geq 0} \varphi\left(X_{n}\right) \geq \delta\right\},
$$

thus, using Doob's inequality, we obtain $V_{\delta}(x) \leq \frac{\varphi(x)}{\delta}$.

Let us again employ excessive functions as in the following definition.

Definition 6. [14, Def.2, p.39] An excessive function $f$ is said to be an excessive majorant of the measurable function $g$ if $f(x) \geq g(x)$ on $\mathscr{X}$. An excessive majorant $f$ of $g$ is said to be the smallest excessive majorant of $g$ if $f(x)$ is less than or equal to any excessive majorant of $g$.

Clearly, $\varphi$ is an SBF of $g$ if and only if $\varphi$ is an excessive majorant of $g$. The following theorem holds over the smallest excessive majorant of a function.

Theorem 7. Let a function $g \in \mathscr{L}$ then the function

$$
\varphi(x)=\lim _{n \rightarrow \infty} \mathscr{Q}^{n} g(x)
$$

is the smallest $S B F$ of the function $g$, i.e. $\varphi$ is an $S B F$ of $g$ and for all $\varphi^{*}$ which is an SBF of $g$ it holds that $\varphi(x) \leq \varphi^{*}(x)$ for all $x \in \mathscr{X}$.

Remark 3. Note that the limit in (19) exists pointwise for all $x \in \mathscr{X}$. Indeed, for any fixed $x$, we have that $\varphi_{n+1}(x) \geq \varphi_{n}(x)$ and due to the monotonicity of the sequence $\left(\varphi_{n}(x)\right)_{n \geq 0}$ the limit $\lim _{n \rightarrow \infty} \varphi_{n}(x)$ always exists, being either finite or infinite. Note also that $\varphi(x)$ defined in (19) is unique.

Alternatively, for a function $g \in \mathscr{L}$, its smallest excessive majorant $\varphi(x)$ satisfies the following equation

$$
\varphi(x)=\max \{g(x), \mathscr{P} \varphi(x)\},
$$

which solution, however, is not unique (see [14, Rem.1, p.43]): if $g(x) \leq C<\infty$ for all $x \in \mathscr{X}$, then for any constant $C^{\prime} \geq C$ the function $\varphi(x) \equiv C^{\prime}$ admits (20).

It is in general difficult to characterize analytically the smallest SBF, as well as to provide strict bounds for the numerical calculations in (19) or (20). On the other hand one can find $V_{\delta}(x)$ using the fact that $V_{\delta}(x)=v\left(x ; \mathscr{X}_{g}(\delta)\right)=1-$ $u\left(x ; \mathscr{X}_{g}^{c}(\delta)\right)$. Notice the similarity of the recurstions in (3) and (19), tailored to calculate respectively $v\left(x ; \mathscr{X}_{g}(\boldsymbol{\delta})\right)$ and $\varphi(x)$. Since the SBF gives only bounds on the reachability probability, the only advantage of characterizing an SBF in the discrete-time case is that, once obtained, it can be applied to any set of the form $\mathscr{X}_{g}(\delta)$, whereas the reachability probability would require being calculated for each of these sets separately,

Let us assume that there exists $\min _{x \in \mathscr{X}} g(x)=\delta_{*}$ and moreover that for all $\delta>\delta_{*}$, we have that $\mathscr{X}_{g}^{c}(\delta)$ is a compact set and $T \in \mathscr{C}_{w}(\mathscr{X})$. By Theorem 3 we know that if there are no absorbing subsets of $\mathscr{X}_{g}^{c}(\boldsymbol{\delta})$ then $u\left(x ; \mathscr{X}_{g}^{c}(\boldsymbol{\delta})\right) \equiv 0$ and the bisimulation is not valid over the infinite horizon. Thus we conclude that to find a finite SBF over an infinite horizon for the processes $X^{1}$ and $X^{2}$ we need for $\mathscr{X}_{g}^{c}(\delta)$ to contain an absorbing subset.

It is interesting to note that also [9] considers cases with the origin as an absorbing set. Due to the monotonicity and compactness of the family $\mathscr{X}_{g}^{c}(\delta)$, that it is equivalent to the following statement: $\mathscr{X}_{g}^{c}(0)=\{x \in \mathscr{X}: g(x)=0\}$ has to contain an absorbing subset. If the function $g$ is locally excessive (i.e. it is excessive on $\mathscr{X}_{g}^{c}(\delta)$ for some $\delta>\delta_{*}$ ) then for the overapproximation of $V_{\delta}(x)$ one can use the decomposition technique provided in Section IV-B.

\section{A. Stochastic Bisimulation Functions: a case study}

We consider a discrete-time approximation of the following stochastic differential equation

$$
d x_{t}=\alpha x_{t} d t+\sigma x_{t} d w_{t},
$$

where we select $\alpha=-1$ and $\sigma=0.3$. The time discretization is obtained by first-order Euler-Maryuama scheme. For two discretization steps $\Delta t$ and $\frac{\Delta t}{2}$ we obtain respectively two discrete time processes, denoted as $X_{n}$ and $X_{n}^{\prime}$. In order to set up a relation over the distance between the two processes, 
we consider $X_{n}$ and $Y_{n}=X_{2 n}^{\prime}$ and select the distance function as follows:

$$
g(x, y)=\left(x^{2}+1\right)\left((x-y)^{2}+1\right)-1 .
$$

In the above expression we employ a distance term between $x$ and $y$, additionally the term $\left(x^{2}+1\right)$ ensures the compactness of sublevel sets, and -1 is used to rescale the minimum of $g$ to be equal to 0 (see discussion above).

The dynamics of the two processes are described by

$$
\begin{aligned}
X_{n+1} & =X_{n}\left(1+\alpha d t+\sigma \xi_{n} \sqrt{d t}\right) \\
Y_{n+1} & =Y_{n}\left(1+\alpha d t+\frac{(\alpha d t)^{2}}{4}+\sigma \sqrt{\frac{d t}{2}}\left(\eta_{n}+\zeta_{n}\right)\right) \\
& +Y_{n}\left(\alpha \sigma \sqrt{\frac{d t^{3}}{8}}\left(\eta_{n}+\zeta_{n}\right)+\sigma^{2} \frac{d t}{2} \eta_{n} \zeta_{n}\right)
\end{aligned}
$$

where $\xi_{n}, \eta_{n}, \zeta_{n}$ are i.i.d. standard normal random variables. Note that the distribution law of $Y_{n+1}$, conditional on $Y_{n}$, is not Gaussian. Two realizations of the processes are plotted in Figure 1.

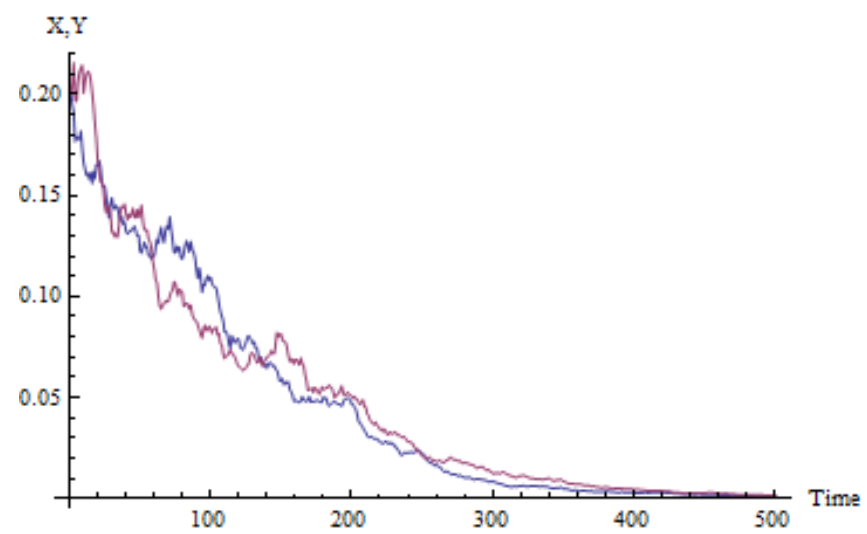

Fig. 1. Realizations of processes $X$ and $Y$ (resp. in red and blue), starting from the initial condition 0.2 .

We select $\Delta t=0.01$ and sets $A=\mathscr{X}_{g}^{c}(0.1)$ and $B=\{x \in$ $\mathscr{X} \mid g(x)<0.01\}$. We would like to compare the values of the quantity $V_{0.1}(0.15,0.15)$ as in $(18)$, obtained by three different techniques: decomposition, direct reachability computation, and via the smallest SBF (through the $\mathscr{Q}$ iterations).

For the computation of the reach-avoid problem (decomposition), of probabilistic reachability, and of the SBF, we have discretized the region $A$ by square cells with diameter equal to 0.015 . With this discretization we can approximate the original processes by a Markov chain [2]. (This also allows computing the associated discretization error, which is not pursued here.) With the Markov chain approximations, we calculate functions $w_{n}, v_{n}$ and $\varphi_{n}$ for $n=2000$ steps. In all these cases the calculation time for the value functions is approximately $109 \mathrm{sec}$.

Notice that there is one absorbing set coinciding with the origin. Hence, the decomposition technique can be used. Using local Doob's inequality we obtain that $\psi(A, B) \geq 0.9$.
Leveraging the decomposition approach, from (11) we obtain the bound $V_{0.1}(0.15,0.15) \leq 0.43$.

The direct calculations of reachability iterations give $V_{0.1}(0.15,0.15) \approx 0.4$. Notice that the straightforward computation does not allow defining a bound.

Finally, the numerical calculation of the smallest SBF $\phi$ by (19) give a very conservative bound: $V_{0.1}(0.15,0.15)<1.04$.

\section{FUTURE WORK}

Current work is focused on the characterization of absorbing sets and on the properties they entail. This is approached from three main directions.

First, the combination of Theorem 3 and Proposition 2 for the Borel state space gives a useful criterion relating absorbance with uniqueness and contractivity of operators $\mathscr{I}_{A}, \mathscr{R}_{A, B}$. Together with the decomposition technique in Theorem 10, it is used to find infinite horizon value functions with strict bounds on the error and to study their properties such as continuity.

Second, the authors are working on the extension of Proposition 3 to the case when the kernels admit densities. For this instance the knowledge of the largest absorbing subset of a given set is extremely useful.

The last direction is to find a way to verify and characterize the absorbing feature of a given set, which is a problem related to other areas of mathematical analysis.

\section{REFERENCES}

[1] A. Abate, S. Amin, M. Prandini, J. Lygeros, and S. Sastry. Probabilistic reachability and safe sets computation for discrete time stochastic hybrid systems. In Proceedings of the 45th IEEE Conference of Decision and Control, pages 258-263, 2006.

[2] A. Abate, J.-P. Katoen, J. Lygeros, and M. Prandini. Approximate model checking of stochastic hybrid systems. European Journal of Control, 16:624-641, December 2010.

[3] A. Abate, J.-P. Katoen, and A. Mereacre. Quantitative automata model checking of autonomous stochastic hybrid systems. In Proceedings of the 14th ACM international conference on Hybrid Systems: Computation and Control, pages 83-92, 2011.

[4] A. Abate, M. Prandini, J. Lygeros, and S. Sastry. Probabilistic reachability and safety for controlled discrete time stochastic hybrid systems. Automatica, 44(11):2724-2734, 2008.

[5] M.L. Bujorianu and J. Lygeros. New insights on stochastic reachability. In Proceedings of the 46th IEEE Conference on Decision and Control, pages 6172-6177, 2007.

[6] R. Durrett. Probability: Theory and Examples - Third Edition. Duxbury Press, 2004.

[7] M. Fränzle, T. Teige, and A. Eggers. Engineering constraint solvers for automatic analysis of probabilistic hybrid automata. Journal of Logic and Algebraic Programming, 79(7):436-466, 2010.

[8] O. Hernández-Lerma and J.B. Lasserre. Discrete-time Markov control processes. Appl. Math, 30, 1996.

[9] A.A. Julius and G.J. Pappas. Approximations of stochastic hybrid systems. Automatic Control, IEEE Transactions on, 54(6):1193-1203, 2009.

[10] H.J. Kushner. Stochastic stability and control, volume 33. Academic Press, 1967.

[11] S.P. Meyn and R.L. Tweedie. Markov chains and stochastic stability. Springer Verlag, 1993

[12] G. Peškir and A. Shiryaev. Optimal stopping and free-boundary problems. Birkhauser, 2006.

[13] F. Ramponi, D. Chatterjee, S. Summers, and J. Lygeros. On the connections between PCTL and dynamic programming. In Proceedings of the 13th ACM international conference on Hybrid Systems: Computation and Control, pages 253-262, 2010.

[14] A. Shiryaev, B. Rozovskii, and G. Grimmett. Optimal stopping rules. Springer-Verlag Berlin Heidelberg, 2008. 